\title{
Comunicación interna en la identidad corporativa de los trabajadores de salud pública en la región Lima
}

\author{
Recibido: 01 de septiembre de 2015 \\ Aceptado: 23 de febrero de 2016 \\ Publicado: 30 de noviembre de 2016
}

\author{
Efraín Ostos Cueva \\ efrain1308@hotmail.com \\ Universidad de San Martín de Porres (Perú)
}

Resumen: La presente investigación tuvo como objetivo principal determinar la relación que existe entre la comunicación interna (CI) como herramienta de Relaciones Públicas (RR. PP.) y la identidad corporativa (IC) de los colaboradores de los establecimientos de salud pública de la región Lima. Es un estudio no experimental con un nivel de investigación correlacional, para lo cual se abordó a una muestra de 224 colaboradores, integrada por trabajadores de una institución de salud. Se pudo demostrar, mediante el coeficiente de correlación de Spearman, que no existe relación significativa entre la CI y la IC de los colaboradores en dicha entidad de salud, así como la evidencia de una relación débil entre las variables de investigación.

Palabras clave: Relaciones Públicas, comunicación interna, estrategias de comunicación, comunicación unidireccional, comunicación bidireccional, identidad corporativa.

\begin{abstract}
The main aim of this research was to determine the relation that exists between the internal communication as tool of Public Relations and the corporate identity of Public Health workers at Lima Region. It's a non-experimental study with a correlational level. The sample was integrated by 224 employees. The research demonstrates (by means of the coefficient of Spearman's correlation) that there is not a significant relation between internal communication and corporate identity in Public Health workers at Lima. Also, there is a weak relation between research variables.
\end{abstract}

Key words: Public Relations, Internal Communication, Communication Strategies, Unidirectional Communication, Bidirectional Communication, Corporate Identity. 


\section{Introducción}

Las acciones de CI se constituyen como una de las actividades más importantes para el desarrollo de toda organización, ya sea pública o privada, permitiendo un entendimiento recíproco entre la institución y sus colaboradores. Asimismo, se yergue como factor vital para la transmisión y consolidación de la IC y contribuye al logro de los objetivos institucionales.

Se sabe que a través de una buena gestión de la CI se logra el entendimiento, confianza y cooperación mutua entre los colaboradores, posibilitando el crecimiento y a la vez posicionamiento de las organizaciones. En ese sentido, resulta necesaria la intervención de las RR. PP. precisamente en la gestión relacional y comunicacional, cuya finalidad es la construcción de buenas relaciones entre la organización y sus públicos.

En el ámbito de las instituciones de salud, Pérez y Solórzano (1999: 239) manifiestan que

“en la problemática total de las comunicaciones aplicadas a nivel de un establecimiento de salud es necesario tener en cuenta movimientos de Relaciones Públicas Internas dirigidas a informar permanentemente al público, aún sobre aspectos que puedan parecer elementales, pero que en el conjunto de un plan orgánico, adquieren incidencia singular".

Es por ello que (a través de un conjunto de acciones comunicativas debidamente dirigidas) se logra obtener una relación que va más allá de aquella vinculada al propio trabajo, donde el colaborador interno se siente satisfecho de la actividad que realiza y, sobre todo, se siente comprometido con el desarrollo de la institución, participando activamente en el progreso de la misma, por medio de actitudes positivas que conllevan a la aportación de sus labores más allá de las que han sido establecidas. De esta manera, se convierte en un transmisor de la imagen de la institución hacia los públicos externos.

Sería en vano esforzarse en desarrollar estrategias de comunicación externa, dejando de lado los problemas humanos existentes dentro de la institución, ya que los colaboradores son el pilar de toda organización; por ello, deben ser reconocidos y atendidos como tal. Frente a esta realidad, la CI juega un papel fundamental en articular este contexto, promoviendo el buen funcionamiento de la institución: al existir buena comunicación en el interior de la organización, se pueden gestionar de manera adecuada los recursos en bien de la misma. Por tal motivo, las acciones de comunicación deben trabajarse primero en casa.

\section{Objetivo y metodología}

El objetivo principal de esta investigación es determinar la relación existente entre las estrategias de CI (sean estas uni y bidireccionales) y la IC (cultura y filosofía corporativa) de los colaboradores de los establecimientos de salud pública de la región Lima. 
El estudio utiliza un diseño de investigación no experimental, ya que no se manipulan las variables, se analiza la realidad tal y como se presenta. El tipo de investigación es aplicativa, ya que hace uso de los conocimientos ya existentes como teorías, enfoques y principios en cada variable de estudio. Asimismo, el nivel de investigación es correlacional, puesto que se establece la relación existente entre la variable CI y la variable IC, explicando por qué ocurre dicho fenómeno y en qué condiciones se da.

La selección de la muestra se determinó por criterios estadísticos de afijación proporcional, arrojando una muestra de 224 colaboradores, utilizándose a la vez el muestreo aleatorio simple. Las dos principales variables de estudio giran en torno a la CI y a la IC. La primera tiene las siguientes dimensiones: estrategias de comunicación, comunicación unidireccional y bidireccional. La segunda variable está integrada por la cultura y la filosofía corporativa.

Con respecto a la recolección de datos, en un principio se realizó la revisión de información bibliográfica, hemerográfica y electrónica respecto a las variables materia de estudio. Luego, la investigación contempló el diseño y aplicación de un instrumento de medición (cuestionario) para conocer la relación existente entre la CI y la construcción de la IC.

\section{La comunicación interna de RR. PP.}

Pérez y Solórzano (1999: 49) manifiestan que "la acción planificada, sistemática y permanente de una organización para armonizar sus intereses con los de sus públicos a través de una actuación aceptable y de una comunicación eficaz, constituye la esencia de las Relaciones Públicas como agente de transformación social”. Por lo tanto, se desprende que la comunicación juega un rol fundamental para la articulación de las acciones de relación con los públicos internos, cuya finalidad es lograr los objetivos institucionales. Sin embargo, se puede apreciar la existencia de debilidades comunicacionales al interior de las organizaciones, donde muchas veces prima el secretismo:

"En las empresas hay una tendencia a no compartir, a no comunicarse, porque erróneamente se piensa que la información es poder; así, algunas personas piensan que retener la información los deja en una posición superior, privilegiada; creen que si sueltan la información se verán en desventaja frente al otro" (De Castro, 2014: 5).

Este tipo de comportamiento genera incertidumbre entre los colaboradores, acción que conlleva a distorsionar la poca información recibida, provocando conflictos que pueden evitarse a través de una correcta gestión de la CI de RR. PP.

Por naturaleza, el ser humano quiere conocer el medio que le rodea. Dentro de las organizaciones, el colaborador siente la necesidad de estar en constante comunicación con su entorno. Pero al escasear dicha comunicación se genera el rumor, consecuencia 
negativa que contamina el ambiente laboral, lo que provoca a la vez una disminución en la productividad, generando al mismo tiempo una falta de IC. En ello radica la importancia de gestionar en forma constante acciones de CI que conlleven a un entendimiento mutuo, donde las RR. PP. se consolidan como un agente transformador al lograr una integración entro los diversos públicos.

Las RR. PP., como agente de transformación social, se convierten así en una filosofía administrativa, puesto que contribuyen a una actitud que ayuda a obtener una eficaz correspondencia e integración con los públicos de una empresa u organización. A la vez, constituyen una tarea comunicativa, por la cual el público toma conocimiento de los propósitos de la empresa, así como esta se entera de las opiniones, actitudes e intereses de aquel, por medio de una información correcta y oportuna. En realidad se genera una reciprocidad en la información y empleo del vehículo comunicativo más adecuado ( $c f$. Pérez y Solórzano, 1999: 61-62).

\subsection{Importancia de la comunicación interna}

Para Ferrari (2007: 96), en el ejercicio de las RR. PP., "la comunicación representa, sin lugar a dudas, el gran valor, puesto que la relación establecida entre las personas y organizaciones ocurre por su uso correcto, en distintas modalidades: interpersonal, dirigida, corporativa, mercadotecnia, etc., y no se limita a los procesos con los medios masivos". Noguero (1990: 110) describe dentro de las funciones de RR. PP. dos aspectos fundamentales:

"Comunicaciones dentro de la empresa. Los Jefes de Departamentos deben ser informados sobre la política de Dirección; los nuevos empleados deben conocer sus responsabilidades y ventajas; vendedores y empleados deben recibir aliento y ayuda; las condiciones internas de trabajo deben ser controladas por un buen equipo de Relaciones Públicas.

Dotar a la entidad de una auténtica filosofía del buen hacer. Es decir, intentar que, en todos los niveles y por todos los medios, se consiga una actitud eficaz y operante ante todos los públicos”.

Por ello, la CI de RR. PP. se constituye como un punto importante para que la organización funcione adecuadamente, ya que sin una gestión adecuada de la CI es imposible que los colaboradores caminen hacia una misma dirección. Si no se gestiona una adecuada comunicación, la institución no podrá alcanzar los objetivos propuestos. Para lograr dichas metas, el relacionista puede hacer uso de una serie de herramientas que permitan un correcto y constante fluido de la comunicación entre todos sus integrantes.

Quienes dirigen las organizaciones deben comprender la importancia que genera la CI, ya que gracias a ella se forja un trabajo en equipo eficiente, logrando obtener una armonía en el centro de labores a través de un flujo de información que permita un entendimiento mutuo. De esta manera, se podrán dar a conocer e interiorizar los valores, objetivos y 
metas que promueve la organización logrando su desarrollo dentro de la sociedad; de lo contrario, al no existir ese entendimiento, la institución no podrá alcanzar a cabalidad sus metas, generando pérdidas para sus directivos. Por ello, la comunicación se manifiesta como sinónimo de progreso, por lo cual debe ir acompañado de un diagnóstico que permita un trabajo comunicacional según la realidad de cada lugar.

\subsubsection{Estrategias de comunicación interna}

Para Matilla (2008: 17-18), la estrategia “está relacionada con una forma de conocimiento encaminada a la búsqueda de la forma más adecuada de satisfacer unos objetivos, a través de la utilización de unos medios. Lo que caracteriza a la estrategia radica en la manera peculiar con que se hace cargo de su objeto -que podríamos denominar 'el decir de un hacer'- y del estilo con que anuncia el designio que preside las mecánicas operativas que dará origen". Según el mencionado autor,

"las estrategias de comunicación son un tipo determinado de estrategias. Una estrategia será comunicativa cuando persiga un objetivo de comunicación, y/o cuando emplee la comunicación para el logro de ese objetivo, en el bien entendido de que la comunicación no es un simple instrumento para alcanzar unos fines, sino un proceso constituyente vital para cualquier organismo" (ibídem: 42).

Por lo tanto, la estrategia de comunicación engloba el conjunto de acciones de comunicación que son elaboradas con anticipación por el relacionista público y su equipo, estableciendo un marco de referencia con la finalidad de lograr los objetivos trazados, teniendo en cuenta las reacciones que causará en cada público. Frente a ello, Salla y Ortega (2008: 35) resaltan la importancia de la labor del relacionista público para la elaboración de las estrategias de comunicación, señalando que

"la planificación estratégica de las relaciones públicas se podría definir como el conjunto de acciones elaboradas por un profesional de las relaciones públicas centradas en el análisis de la situación de la organización a la que sirve, su entorno, los públicos con los que se relaciona y los recursos disponibles convenientemente optimizados para determinar las líneas de actuación que permita el logro de los objetivos y metas en base a unas estrategias que se correspondan con las políticas de la empresa”.

Al mismo tiempo, Matilla (2008: 45) sostiene que

"la planificación estratégica se constituye en una función básica de la dirección de la comunicación en las organizaciones con sus públicos y con la opinión pública, en tanto acto de pensamiento, ya que es un proceso racional, lógico y político que supone llevar a cabo estudios, plantearse preguntas, definir diagnósticos, tomar decisiones, establecer objetivos a lograr, disponer recursos, implementar acciones". 
De lo expuesto, se desprende que la planificación estratégica no son acciones que se organizan de la noche a la mañana (no es una varita mágica) para solucionar un problema en forma inmediata. La planificación estratégica se constituye como un instrumento que forma parte de las actividades de RR. PP., permitiendo de esta manera erradicar la improvisación de actividades, logrando los efectos realmente deseados. Sin una planificación estratégica no se podrán desarrollar las actividades de comunicación ni tender puentes que permitan un mejor flujo comunicacional entre la organización y los colaboradores.

La estrategia nace para la acción, ya que debe ser ejecutada, de modo tal que sin planificación la estrategia no sería más que un mero proyecto. Por consiguiente, para convertir la estrategia en realidad se precisa de un ordenamiento de las actividades, asignándoles prioridades de valor (que definan qué es lo importante) y de tiempo (qué debe ejecutarse en primer lugar). Pero, además, la planificación coadyuvará a estudiar y cifrar más rápidamente la previsión de las consecuencias que pueda ocasionar cualquier movimiento estratégico (ibídem: 53).

Las estrategias de comunicación que emprenda la organización deben estar alineadas con aquello que desea la institución y aquello que los colaboradores buscan:

"Las estrategias comunicacionales deben apuntar hacia el público interno y externo; las expectativas de las empresas como de los colaboradores deben estar alineadas. El atraer y retener al mejor talento del mercado sirve para mantener la ventaja competitiva. El papel de los públicos internos se volvió -más que nunca- primordial, todos deben conocer la estrategia y saber qué deben hacer desde sus respectivos puestos de trabajo para sostenerla y fortalecerla. En la gran mayoría de los casos, una mala ejecución, y no una mala estrategia, es la causa de los fracasos.

Por estos motivos, los primeros voceros de la empresa son los colaboradores. Cuando ellos están convencidos de un ideal, de una meta y conocen el camino para llegar a ella, su accionar, satisfacción laboral y convicción maximizará las relaciones de colaboración" (Alcívar, 2013: 95).

Del mismo modo, se menciona que "la estrategia es el camino para posicionar un concepto, sugiere el conjunto de acciones organizadas y de un trabajo del intelecto creativo. Su aplicación requiere de la planeación, dirección, ejecución, seguimiento y evaluación de cada acción estratégica. Sin embargo, es necesario considerar la oportunidad del momento, los recursos y el espacio de implementación" (López Jiménez y otros, 2007: 33). Por lo tanto, la estrategia estará determinada por el conjunto de tácticas que se desarrollarán con el objetivo de alcanzar las metas.

La estrategia radica en la planificación general que determina la naturaleza y los principios generales que habrá que aplicar en las acciones de comunicación dirigidas al público objetivo, definiendo los medios que habrá que utilizar para llegar a los resultados propuestos ( $c f$. Marín, 2009: 44). Siguiendo esta línea, se menciona que las estrategias de comunicación vienen a representar "la suma de las tácticas, entendidas estas como el 
sumatorio de las decisiones singulares que constituyen la cadena de decisiones. Y pueden también definirse como el método o conjunto de métodos seguidos por el comunicador para seleccionar, estructurar y difundir su comunicación para alcanzar los objetivos asignados, siempre teniendo en cuenta las posibles reacciones de los demás y de los cambios del entorno" (Matilla, 2008: 42).

La táctica ayuda a dar un orden a los recursos existentes en busca de un determinado fin, reduciendo así el margen de error, logrando minimizar las acciones espontáneas o repetir aquello que ya se puso en práctica y no funcionó. Dentro de las tácticas, Di Génova (2012: 74), destaca acciones aplicadas al público interno:

- Revista interna: conteniendo información de interés exclusivo para el público interno, el cual se debe realizar con una determinada periodicidad.

- Carteles: con información más frecuente y sobre todo dinámica que debe ser de rápido acceso.

- Capacitaciones: fortaleciendo los conocimientos, capacidades, actitudes y destrezas de los colaboradores.

- Manual del empleado: conteniendo información para la etapa de inducción para el personal, normas y disposiciones internas, filosofía y objetivos de la organización.

- Buzón de sugerencias para la comunicación interna: habilitando un canal de información desde el personal de base hasta el nivel más alto, propiciando un espacio de participación que permita motivar al colaborador desarrollando un sentido de pertenencia.

- Programa de beneficios y fidelización para públicos internos: implementar acciones tendientes a generar satisfacción laboral a través de una premiación y/o reconocimiento.

\subsubsection{Comunicación unidireccional}

Para Martínez Guillén (2012: 7), "la comunicación unidireccional es aquella en que la información fluye en una sola dirección, de manera que la recepción del feedback (retroalimentación o información que recibe el receptor) está limitada. Por lo tanto, la comunicación unidireccional es aquel tipo de comunicación dirigida en un solo sentido, es decir, es el proceso mediante el cual una persona expone una idea a otra $u$ otras personas, quienes lo reciben, pero no existe una respuesta directa.

Este tipo de comunicación es muy común en las organizaciones, donde las autoridades son los únicos encargados de dirigir la comunicación, informando a los subordinados sobre las acciones a emprender, sin que los colaboradores puedan expresar si han comprendido o no la información recibida.

Los programas de comunicación interna han dado prioridad a un grupo de técnicas de comunicación unidireccional de los directivos a los empleados, con el principal objetivo de diseminar la información, transmitir decisiones y controlar a la gente. Esta noción tiene un carácter claramente informacional, porque se centra en informar a los empleados sobre noticias y actividades que están ocurriendo en la organización (Carretón y Ramos, 2009: 43). 
Este tipo de comunicación tiene como ventaja controlar la información a través de una autoridad, donde no existe una respuesta de quien o a quienes va dirigido el mensaje, lo que a la vez conlleva a una pérdida de oportunidades de comunicación, generando inseguridad y desconfianza en torno a la información.

\subsubsection{Comunicación bidireccional}

Es el tipo de comunicación donde el emisor y el receptor intercambian información como consecuencia de un entendimiento mutuo. Este tipo de comunicación permite un intercambio de mensajes precisando la información recibida $\mathrm{y}$, a la vez, permite la aclaración y comprobación inmediata de lo comunicado, resultando más eficaz que la comunicación unidireccional.

En la comunicación bidireccional, la información emitida se va ajustando a las condiciones del receptor a medida que este va interviniendo y dando sus opiniones. Estas opiniones se pueden expresar verbalmente, gestualmente o en forma escrita. La comunicación no puede ser eficaz si no cuenta con la participación interesada de todos los interlocutores (Martínez Guillén, 2003: 9). Bien se sabe que toda organización está compuesta por un grupo humano, quienes interactúan en forma constante para poder alcanzar los objetivos institucionales. Frente a ello, la comunicación bidireccional juega un rol fundamental al aumentar la comprensión y promover el entendimiento entre los colaboradores y la gerencia.

De Castro (2014: 92) manifiesta que la comunicación bidireccional se da "con el control del director y con participación del equipo de trabajo. Este es el tipo de reunión más importante, ya que le permite al grupo una mejor comprensión de los problemas que se discuten y se logra una mayor aceptación de las ideas expuestas y de las decisiones tomadas, debido a que el mismo grupo ha participado de estas".

\subsection{Identidad corporativa}

Capriotti (2009: 21) la define "como el conjunto de características centrales, perdurables y distintivas de una organización, con las que la propia organización se autoidentifica (a nivel introspectivo) y se autodiferencia (de las otras organizaciones de su entorno)". Por lo tanto, cada organización es distinta a la otra, así sean del mismo rubro (instituciones de salud pública) siempre existirán diferencias, cada entidad tendrá un punto en particular.

A la vez, el mencionado autor manifiesta que la IC viene a representar la personalidad de la organización, lo que ella es y pretende ser. Incluye su ser histórico, ético y de comportamiento, aquello que la hace individual y la distingue y diferencia de las demás organizaciones (ápud Martín, 2005: 54).

Frente a ello, se puede mencionar que la IC representa el conjunto de acciones que son arrastradas a lo largo del tiempo, imprimiendo un sello particular que los distinguirá de los demás. Por otra parte, Costa (2012: 86) describe a la IC como "el ADN de la empresa, 
donde están inscritas las claves de su singularidad, su capacidad, la aptitud adaptativa que asegura su autoequilibrio, y el potencial de su desarrollo futuro".

A la vez sostiene que "la IC es un sistema de signos visuales que tiene por objeto distinguir -facilitar el reconocimiento y la recordación- a una empresa u organización de las demás. $\mathrm{Su}$ misión es, pues, diferenciar, asociar ciertos signos con determinada organización y significar, es decir, transmitir elementos de sentido, connotaciones positivas; en otras palabras, aumentar la notoriedad de la empresa (Costa, 1993: 15).

Consecuentemente, toda institución maneja una determinada identidad, sin ella no puede existir, ya que viene a representar la razón de ser de dicha organización. Por lo tanto, es el factor o pilar indispensable que da vida a una determinada entidad, la que debe calar en la mente de los públicos. Para Villafañe (2008: 26), adoptando el término de identidad de la empresa, la define como "el conjunto de rasgos y atributos que definen su esencia, algunos de los cuales son visibles y otros no".

Por consiguiente, la IC incluye una serie de atributos propios, los cuales deben ser expresados de forma visible y a la vez debe estar interiorizado en cada miembro de la organización para luego transmitirlo en forma verbal y no verbal. Además, Argüello (2005: 9) señala que la IC "es la percepción de autenticidad que tiene la organización de sí misma, condicionada por el entorno, su propia actividad identitaria y el proceso continuo de revisión y creación de sus atributos, de su cultura, lo cual debe comunicar a sus audiencias, a su público".

Para la formación de la IC se requiere entre otras cosas una serie de acciones de comunicación interna, que proyecte significados comunes entre todos los colaboradores, permitiendo establecer una relación de identidad con la organización, lo que conlleva al cumplimiento adecuado de su trabajo, ya que a través de interacciones comunicativas que promueva la institución se logra un sentido de pertenencia y compromiso entre sus integrantes.

La IC está integrada por dos componentes esenciales: la cultura corporativa y la filosofía corporativa ( $c f$. Capriotti, 2009: 23) que se detallarán en los dos siguientes subapartados.

\subsubsection{Cultura corporativa}

Está constituida por el conjunto de normas, valores y pautas de conducta que son compartidos por los integrantes de la organización, la cual se ve reflejada en su comportamiento. Estas acciones son determinadas por factores como la personalidad de los directivos y los colaboradores, por la historia de la institución, el entorno social donde se desenvuelve, entre otros ( $c f$. Ronnie, 2007: 2).

La cultura viene a ser un factor de integración, ya que los miembros de la organización para sentirse parte de ella, deben cumplir con las pautas previamente establecidas. Dentro de los componentes de la cultura corporativa tenemos: 
- Creencias: viene a ser el conjunto de presunciones básicas compartidas por los miembros de la organización sobre los diferentes aspectos y cuestiones de la entidad (cf. Capriotti, 2009: 24). Son estructuras invisibles, inconscientes y asumidas como preestablecidas.

- Valores: es el conjunto de principios éticos que son compartidos por los integrantes de la organización, permitiendo crear pautas de comportamiento que deben ser contrastados con la realidad.

- Pautas de conducta: son modelos de comportamiento que pueden ser observados, e indican como se desarrolla la cultura corporativa expresada a través de las creencias y valores de la organización.

Por tanto, la cultura corporativa viene a representar un elemento importante para la organización, ya que a través de las creencias, valores y pautas de conducta que envuelven a la institución, influirá en la valoración que tengan los colaboradores de su centro de labores.

\subsubsection{Filosofía corporativa}

Identifica la forma de ser de la organización, es el conjunto de principios sobre los cuales se sustenta la institución, lo cual define el carácter de la entidad, creando un sentido de identidad y pertenencia, conllevando a un comportamiento ético y de consenso, con el objetivo de alcanzar las metas trazadas. La filosofía corporativa es establecida por la alta dirección y ha de ser el signo distintivo de la organización, ya que representa las creencias, valores y pautas de conducta de sus integrantes (Capriotti, 2009: 25). Sus componentes son los siguientes:

- Misión: define la actividad que desarrolla la organización, es lo que hace la institución. Por lo tanto, es el motivo, razón o fin de la existencia de una organización. "Una declaración de misión bien pensada proporciona a los empleados un sentido común de propósito, rumbo y oportunidad. También guía a empleados dispersos geográficamente para que trabajen con independencia pero en conjunto hacia el logro de las metas de la organización" (Kotler, 2002: 41).

- Visión: es la representación clara que indica el norte de la organización a largo plazo; por lo tanto, es la perspectiva de futuro de la institución, en ella se indica a donde se quiere llegar, viene a representar el reto particular de la organización, "lo que aspiramos ser; hacia donde vamos con nuestro emprendimiento; la promesa hacia nosotros, nuestro personal, nuestros clientes y nuestros proveedores" (Spina, 2006: 51).

- Valores: son los principios éticos sobre los cuales se desenvuelve la organización, permitiendo la generación de pautas de conducta entre sus integrantes. Sanz y Gonzáles (2005: 66), mencionan que se debe "considerar a los valores como conformadores de 
actitudes, pero también como prescriptores del comportamiento humano, por cuanto conforman una estructura de tipo cognitivo que sirve al individuo para interpretar y orientarse en su entorno social".

\section{Comunicación interna en las instituciones de salud}

Al igual que en otras organizaciones, busca la transmisión de mensajes a través de estrategias dirigidas al público interno, a fin de optimizar la gestión del capital humano y mejorar la labor de los servicios sanitarios. Como lo menciona Medina (2012: 19), "la CI constituye una verdadera herramienta directiva ya que afecta a todos los empleados del hospital e influye en el funcionamiento de la institución, en la calidad del servicio ofrecido al paciente y en la imagen de marca del hospital".

Al gestionar la CI se logra una circulación adecuada de la comunicación, permitiendo que la alta dirección obtenga información necesaria para mejorar sus procesos, facilitando a la vez coordinación y cohesión entre todos los colaboradores, generando en el personal un sentido de pertenencia (identidad) hacia la organización, para que después sean los propios trabajadores quienes puedan transmitir la cultura de la institución a los públicos externos (usuarios).

La CI no queda limitada al interior de la organización, sino que los resultados de una buena CI se reflejan en las relaciones externas y, en este sentido, repercute en la imagen externa de la institución. En el caso del hospital, los usuarios están en contacto con los profesionales sanitarios y no sanitarios de manera constante, por lo que los empleados se convierten en los principales embajadores (o representantes) de la institución por la cual trabajan. Por eso se considera que al medir la satisfacción laboral de los trabajadores del hospital, se está midiendo una parte esencial de la calidad del servicio prestado (Costa, 2011: 96).

Por consiguiente, la CI bien dirigida proporciona una relación de motivación y compromiso de los colaboradores, lo que permite a la vez incrementar la calidad de atención percibida por los usuarios y su nivel de satisfacción respecto al servicio prestado. Frente a ello, Medina (2012: 23) señala que "la comunicación interpersonal entre los empleados del hospital constituye una de las acciones de comunicación interna más importante que tienen lugar en el hospital. En segundo lugar, la comunicación influye en la satisfacción del paciente". Así, la CI busca la circulación de los mensajes en el momento adecuado, no antes ni después,

"trata de contar a los clientes internos lo que la propia organización está haciendo, permitiendo construir una identidad de la empresa susceptible de conciliar entre sí al conjunto del personal y crear un clima de confianza y motivación. De ese modo puede construirse un contrato que podríamos calificar de 'emocional' con los objetivos de la organización, entre la alta dirección y los clientes internos” (Menéndez y Vadillo, 2009: 25). 
En el Perú, el Ministerio de Salud (MINSA), como ente rector de las políticas comunicacionales de dicho sector, es el encargado de la elaboración de normas, guías, documentos o manuales que sirven de referencia a las diversas instituciones de salud pública, lo que conlleva a un adecuado manejo de la comunicación con sus colaboradores internos, es decir, entre el personal médico, de enfermería, obstetricia, técnico asistencial y administrativo. Por consiguiente, aquella información sobre una determinada situación dentro del sector salud (que puede desencadenar en una crisis) llega a través de los propios colaboradores internos:

"Cuando la institución cuenta con un buen sistema de comunicación interna y fortalece sus capacidades corporativas, la recepción y el manejo de información es mayor y rápida. Ello es importante si se tiene en cuenta que el tiempo es uno de los factores críticos en el manejo o la gestión de crisis" (MINSA, 2006: 14).

Para poder responder de manera adecuada a la demanda de información, los responsables del área de RR. PP. procesan los argumentos técnico-médicos hacia formatos más didácticos y sencillos, clarificando ideas y respuestas. Además, los relacionistas públicos convierten las situaciones de crisis en oportunidades para identificar debilidades y el fortalecimiento de las mismas, liderando el equipo. De esta manera, se planifican las estrategias para un mejor manejo de la información (ibídem).

\section{Resultados}

Los datos (recogidos de la aplicación del instrumento de investigación y el análisis de los mismos) se han obtenido con la finalidad de dar respuesta a las interrogantes planteadas en el presente trabajo (figuras 1-9).

Figura 1: ¿Las reuniones informativas en las que has participado, ha logrado que conozcas mejor a la institución?

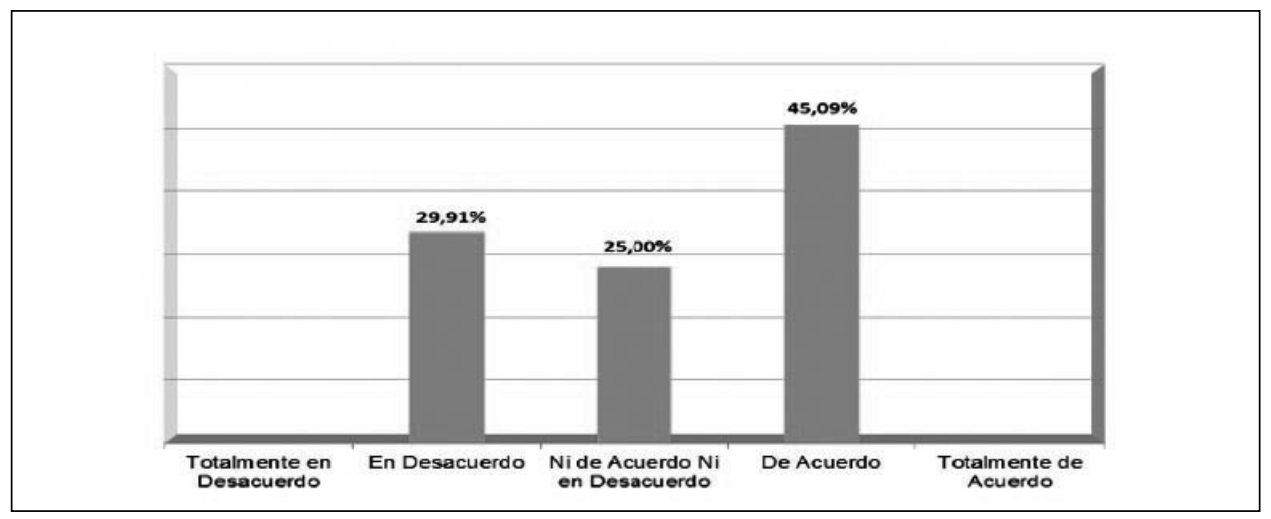

Fuente: Elaboración propia. 
A la pregunta mencionada (figura 1), el $45.09 \%$ considera que las reuniones informativas permiten conocer mejor a la institución, mientras que el $29.91 \%$ está en desacuerdo con la premisa. El 25\% no estaba de acuerdo ni en desacuerdo.

Figura 2: Cuándo las autoridades se dirigen a los colaboradores, ¿utilizan palabras que son de fácil entendimiento?

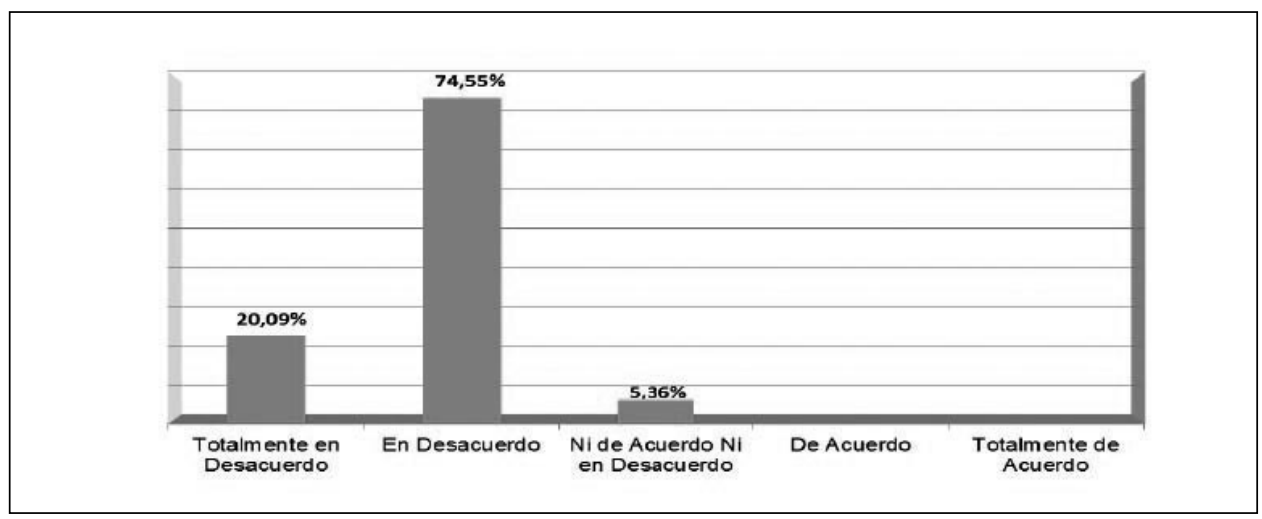

Fuente: Elaboración propia.

A la pregunta citada (figura 2), el $74.55 \%$ de los encuestados está en desacuerdo con la premisa, manifestando que las autoridades no utilizan palabras de fácil entendimiento al dirigirse a los colaboradores. El $20.09 \%$ está totalmente en desacuerdo y el $5.36 \%$ respondió que no estaba de acuerdo ni en desacuerdo.

Figura 3: ¿Consideras que existen canales de comunicación que transmiten tus comentarios o dudas a las autoridades?

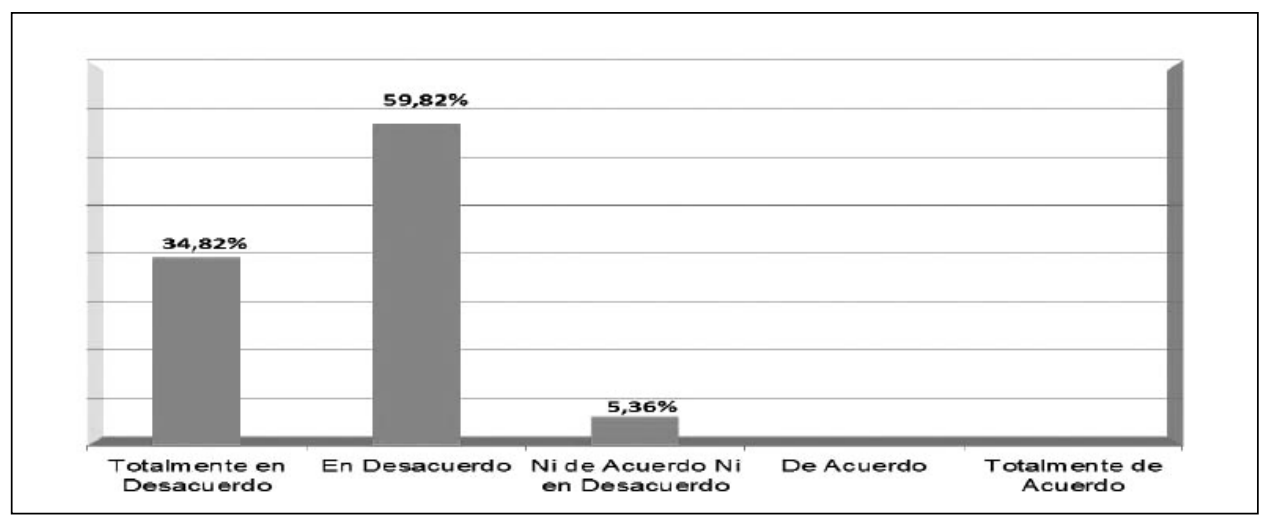

Fuente: Elaboración propia.

A la pregunta citada (figura 3), el 59.82\% de los encuestados está en desacuerdo, considerando que no existen canales de comunicación que transmitan comentarios o dudas 
a las autoridades. El 34.82\% está totalmente en desacuerdo y el 5.36\% respondió que no estaba de acuerdo ni en desacuerdo.

Figura 4: ¿Conoces que medios o canales de comunicación puedes utilizar para que un comentario llegue hasta la persona responsable?

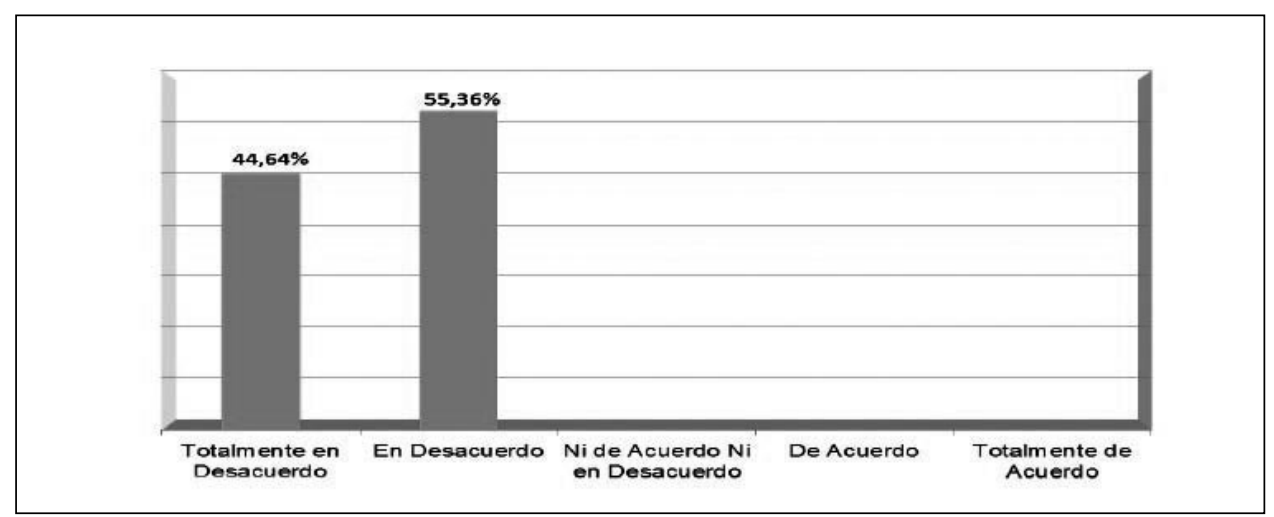

Fuente: Elaboración propia.

A la pregunta mencionada (figura 4), el 55.36\% de los encuestados está en desacuerdo, es decir, no conoce que medio o canal de comunicación puede utilizar para hacer llegar un comentario a un superior, mientras que el $44.64 \%$ está totalmente en desacuerdo.

Figura 5: ¿Consideras que la organización comunica

los valores institucionales a los colaboradores?

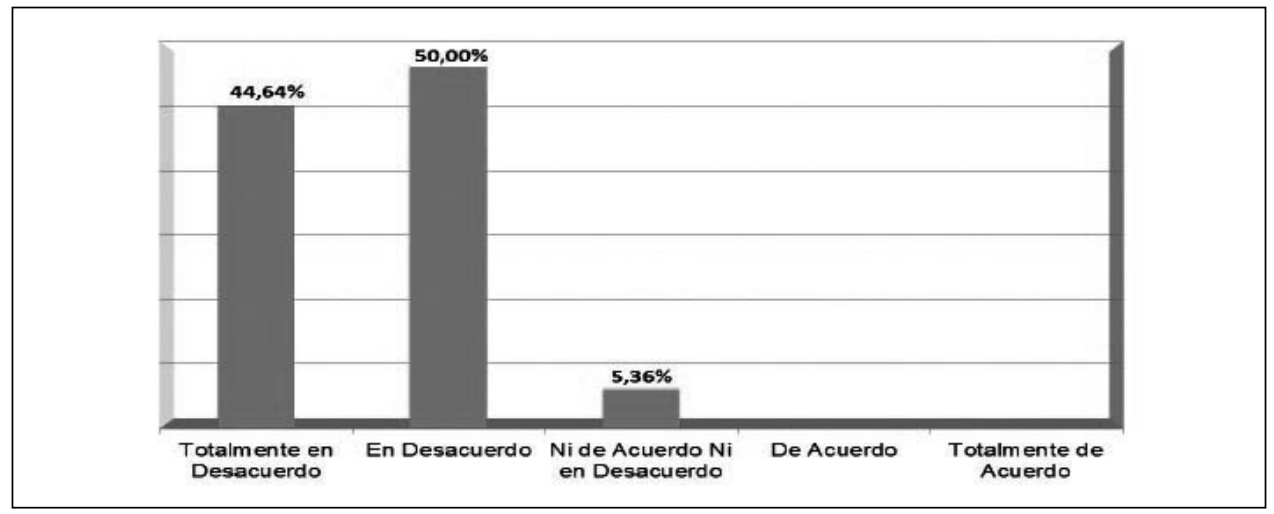

Fuente: Elaboración propia.

A la pregunta citada (figura 5), el 50\% de los encuestados está en desacuerdo, considerando que la organización no comunica los valores que la rigen, el 44.64\% está totalmente en desacuerdo y el 5.36\% respondió que no estaba de acuerdo ni en está desacuerdo. 
Figura 6: ¿Consideras que la organización difunde las normas de conducta que debe adoptar el colaborador durante el desempeño de sus funciones?

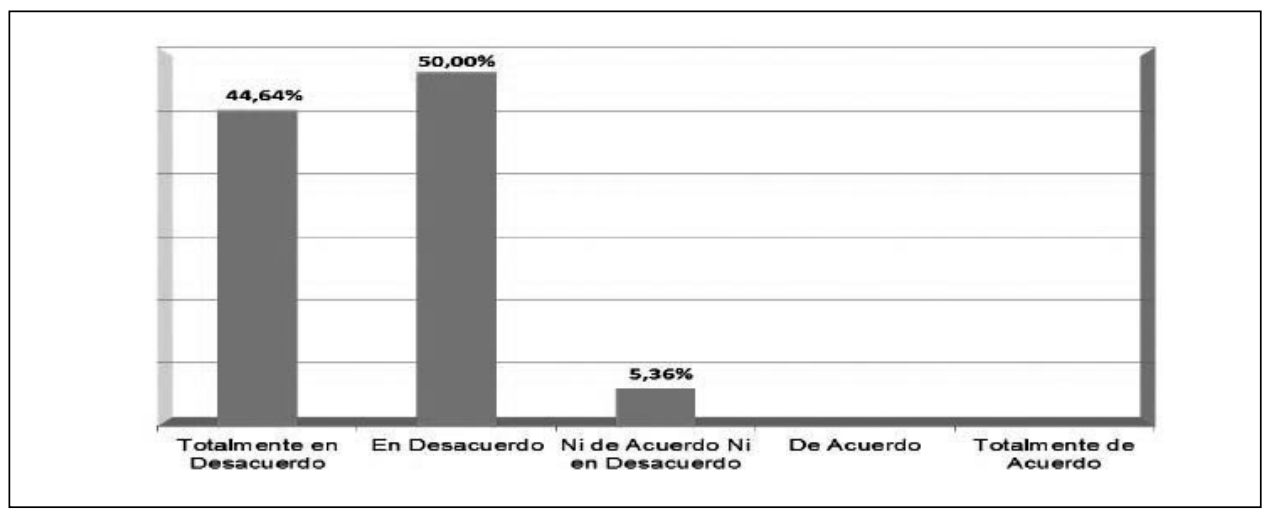

Fuente: Elaboración propia.

A la pregunta citada (figura 6), el 50\% de los encuestados está en desacuerdo, manifestando que la organización no difunde las normas de conducta, el $44.64 \%$ está totalmente en desacuerdo, y el 5.36\% respondió que no estaba de acuerdo ni en desacuerdo.

Figura 7: ¿Recuerdas cuál es la visión de la institución?

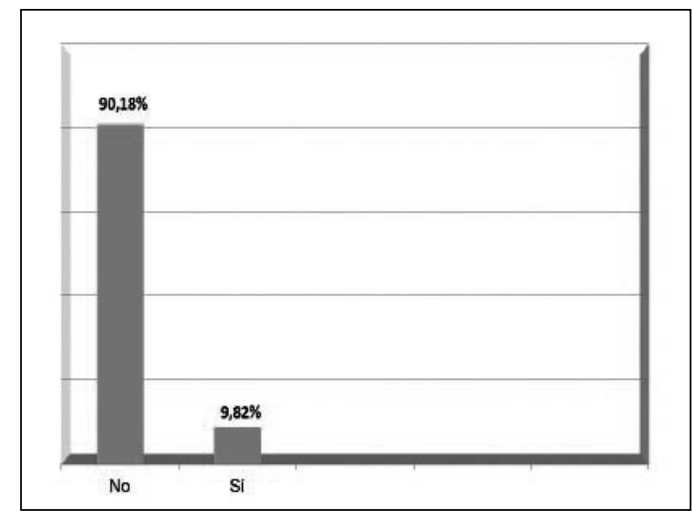

Fuente: Elaboración propia.

A la pregunta planteada (figura 7), el 90.18\% de los encuestados no recuerda la visión de la institución, sólo el $9.82 \%$ lo recuerda. 
Figura 8: ¿Recuerdas cuál es la misión de la institución?

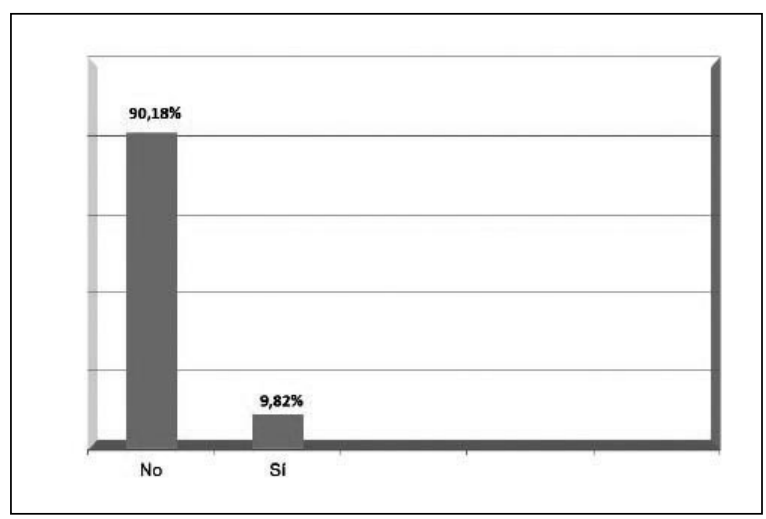

Fuente: Elaboración propia.

A la pregunta planteada (figura 8), el 90.18\% de los encuestados no recuerda la misión de la institución. Sólo el $9.82 \%$ lo recuerda.

Figura 9: ¿Recuerdas algún objetivo institucional?

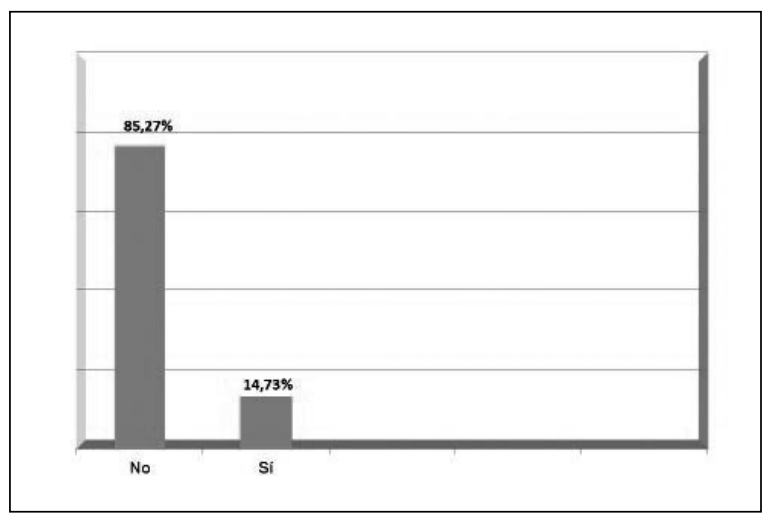

Fuente: Elaboración propia.

A la pregunta planteada (figura 9), el $85.27 \%$ de los encuestados no recuerda ninguno de los objetivos institucionales, solo el $14.73 \%$ recuerda alguno de ellos.

\section{Conclusiones}

Las principales conclusiones de esta investigación han sido extraídas de cada una de las mediciones realizadas con respecto a la relación que existe entre la CI y la IC de los colaboradores de los establecimientos de salud pública de la región Lima. Frente a esta premisa, se puede mencionar que toda organización tiene la necesidad de hacer uso de la comunicación interna, 
pero para ello es necesario que exista una evaluación y selección de mensajes y medios que se vayan a utilizar con el objetivo de comunicar y/o fortalecer la identidad corporativa en sus colaboradores; en diversas investigaciones se menciona que muchos de los problemas que se suscitan al interior de las instituciones están relacionadas con la falta de comunicación.

Tomándose en cuenta a la organización materia de estudio, se aprecia que existen canales de comunicación conocidos por los colaboradores. Sin embargo, estos muestran falencias, no logrando los resultados esperados, volviéndose ineficaz para que los integrantes de la entidad obtengan de primera mano información importante y necesaria para conocer cual es la visión, misión, objetivos y metas a alcanzar.

Se ha determinado que las actividades de CI no se relacionan de manera significativa con la IC de los colaboradores de los establecimientos de salud pública de la región Lima. La comprobación de esta hipótesis general fue realizada aplicando la prueba no paramétrica del coeficiente de correlación de Spearman, teniendo como resultado $\mathrm{rs}=0.416$ y $\mathrm{p}=0<0.05$.

Frente a las estrategias de CI, se ha determinado que existe una relación negativa entre dichas estrategias y la cultura corporativa de los colaboradores de los establecimientos de salud pública de la región Lima. La comprobación de esta hipótesis específica fue realizada aplicando la prueba no paramétrica del coeficiente de correlación de Spearman, teniendo como resultado $\mathrm{rs}=-0.009$ y $\mathrm{p}=0.897>0.05$.

Se ha podido determinar que las estrategias de CI no tienen relación significativa con la filosofía corporativa de los colaboradores de los establecimientos de salud pública de la región Lima. La comprobación de la hipótesis específica fue realizada aplicando la prueba no paramétrica del coeficiente de correlación de Spearman, teniendo como resultado $\mathrm{rs}=$ -0.131, y $\mathrm{p}=0.05>0.05$.

Se determinó que la comunicación unidireccional interna no tiene relación significativa con la cultura corporativa de los colaboradores de los establecimientos de salud pública de la región Lima. La comprobación de la hipótesis específica fue realizada aplicando la prueba no paramétrica del coeficiente de correlación de Spearman, teniendo como resultado rs $=$ 0.610, y $\mathrm{p}=0<0.05$.

Se comprobó que existe relación negativa entre la comunicación unidireccional interna y la filosofía corporativa de los colaboradores de los establecimientos de salud pública de la región Lima. La comprobación de esta la hipótesis específica fue realizada aplicando la prueba no paramétrica del coeficiente de correlación de Spearman, teniendo como resultado $\mathrm{rs}=-0,112, \mathrm{y} \mathrm{p}=0,096>0.05$.

Se pudo corroborar que no existe relación significativa entre la comunicación bidireccional interna y la cultura corporativa de los colaboradores de los establecimientos de salud pública de la región Lima. La comprobación de esta hipótesis específica fue realizada aplicando la prueba no paramétrica del coeficiente de correlación de Spearman, teniendo como resultado $\mathrm{rs}=0,788, \mathrm{y} \mathrm{p}=0<0.05$. 
Se determinó que no existe relación significativa entre la comunicación bidireccional interna y la filosofía corporativa de los colaboradores de los establecimientos de salud pública de la región Lima. La comprobación de la hipótesis específica fue realizada aplicando la prueba no paramétrica del coeficiente de correlación de Spearman teniendo como resultado $\mathrm{rs}=0.292, \mathrm{y} \mathrm{p}=0<0.05$.

\section{Fuentes consultadas}

Alcívar, G. (2013). "Los pilares fundamentales de la formación académica del nuevo relacionista público". Revista ALACAURP, núm. 7, pp. 91-97. Extraída el 12/V/2016 desde http://alacaurp.org/pdf/Revista_VII-VII_Congreso_Internacional_ALACAURP.pdf

Argüello, J. (2005). Identidad e imagen corporativa. Buenos Aires: El Cid Editor.

Boiry, P. (1998). Relaciones Públicas o la estrategia de la confianza. Barcelona: Gestión 2000.

Capriotti, P. (2009). La imagen de empresa. Estrategia para una comunicación integrada. Madrid: Ateneo.

Carretón, B. y Ramos, I. (2009). Las Relaciones Públicas en la gestión de la comunicación interna. Sevilla: AIRP.

Costa, C. (2011). La comunicación en el hospital. La gestión de la comunicación en el ámbito sanitario. Sevilla: Comunicación Social.

\section{Costa, J.}

_(2012). Dirección y gestión de la comunicación en la nueva economía. Barcelona: CPC. _(1993). Identidad corporativa. México D. F.: Trillas.

De Castro, A. (2014). Comunicación organizacional, técnicas y estrategias. Barranquilla: Universidad del Norte.

Di Génova, A. (2012). Manual de Relaciones Públicas e institucionales. Estrategias de comunicación y tácticas relacionales. Buenos Aires: Ugerman.

Grunig, J. y Hunt, T. (2003). Dirección de Relaciones Públicas. Barcelona: Gestión 2000.

Ferrari, M. (2007). "La comunicación como apoyo estratégico en la empresa". Revista ALACAURP, núm. 4, pp. 140-153.

Hernández, R.; Fernández, C. y Baptista, P. (1999). Metodología de la investigación. México D. F.: McGraw-Hill. 
Kotler, P. (2002). Dirección de marketing. Conceptos esenciales. México D. F.: Pearson.

López Jiménez, D. y otros (2007). Comunicación empresarial. Plan estratégico como herramienta gerencial. Bogotá: Ecoe Ediciones.

Marín, F. (2009). Comunicación de crisis. Madrid: LID.

Martín, M. (2005). Arquitectura de marcas, modelo general de construcción de marcas y gestión de sus activos. Madrid: Editorial ESIC.

\section{Martínez Guillén, M.}

_(2012). La comunicación en la empresa. Madrid: Ediciones Díaz de Santos.

_(2003). La gestión empresarial, equilibrando objetivos y valores. Madrid: Ediciones Díaz de Santos.

Matilla, K. (2008). Los modelos de planificación estratégica en la teoría de las Relaciones Públicas. Barcelona: Editorial UOC.

Medina, P. (2012). "El valor estratégico de la comunicación interna hospitalaria". Revista de Comunicación y Salud. Vol. 2, núm. 1, pp. 19-28. Extraída el 12/V/2016 desde http://www.revistadecomunicacionysalud.org/index.php/rcys/article/view/26

Menéndez, D. y Vadillo, F. (2009). El plan de comunicación hospitalario: herramienta de gestión sanitaria. Alicante: Club Universitario.

MINSA, Ministerio de Salud del Perú (2006). "Manual para el manejo de la comunicación en situaciones de crisis". Extraída el 12/V/2016 desde ftp://ftp2.minsa.gob.pe/docconsulta/documentos/ogc/GUIAINTERVENCIONENCRISIS/ MANUALCRISISJULIO2006OK.pdf

Noguero, A. (1990). Relaciones Públicas e industria de la persuasión. Análisis gnoseológico y situacional. Barcelona: Promociones y Publicaciones Universitarias.

Pérez, R. y Solórzano, E. (1999). Relaciones Públicas Superiores, una nueva pedagogía. Lima: USMP.

Ronnie, L. (2007). Gestión de la cultura corporativa. Madrid: Ediciones Díaz de Santos.

Salla, J. y Ortega, J. (2008). Plan estratégico de Relaciones Públicas. Barcelona: Bosch Empresa.

Sanz, M. y Gonzáles, M. (2005). Identidad Corporativa, claves de la comunicación empresarial. Madrid: ESIC.

Solórzano, E. (2000). "Relación humana, legitimidad y acción comunicacional”. Revista ALACAURP, núm. 1, pp. 5-11. 
Spina, M. (2006). Guía práctica para emprender en el diseño. Buenos Aires: CommTOOLS.

Stephenson, H. (1960). Relaciones Públicas. Lima: PUCP.

Villafañe, J. (2008). Imagen positiva, gestión estratégica de la imagen de la empresa. Madrid: Ediciones pirámide.

Xifra, J. (2010). Relaciones Públicas, empresa y sociedad. Una aproximación ética. Barcelona: UOC. 